\title{
A Functional Magnetic Resonance Imaging Study on the Neural Mechanisms of Hyperalgesic Nocebo Effect
}

\author{
Jian Kong, ${ }^{1,2}$ Randy L. Gollub, ${ }^{1,2,3}$ Ginger Polich, ${ }^{1}$ Irving Kirsch, ${ }^{4}$ Peter LaViolette, ${ }^{1,2}$ Mark Vangel, ${ }^{2,3}$ Bruce Rosen, ${ }^{2}$ and \\ Ted J. Kaptchuk ${ }^{5}$ \\ ${ }^{1}$ Department of Psychiatry, Massachusetts General Hospital, ${ }^{2}$ Massachusetts General Hospital/Massachusetts Institute of Technology/Harvard Medical \\ School Athinoula A. Martinos Center for Biomedical Imaging, and ${ }^{3}$ Massachusetts General Hospital Clinical Research Center Biomedical Imaging Core, \\ Charlestown, Massachusetts 02129, ${ }^{4}$ Department of Psychology, University of Hull, Hull HU6 7RX, United Kingdom, and ${ }^{5}$ Osher Institute, Harvard Medical \\ School, Boston, Massachusetts 02215
}

Previous studies suggest that nocebo effects, sometimes termed "negative placebo effects," can contribute appreciably to a variety of medical symptoms and adverse events in clinical trials and medical care. In this study, using a within-subject design, we combined functional magnetic resonance imaging (fMRI) and an expectation/conditioning manipulation model to investigate the neural substrates of nocebo hyperalgesia using heat pain on the right forearm. Thirteen subjects completed the study. Results showed that, after administering inert treatment, subjective pain intensity ratings increased significantly more on nocebo regions compared with the control regions in which no expectancy/conditioning manipulation was performed. fMRI analysis of hyperalgesic nocebo responses to identical calibrated noxious stimuli showed signal increases in brain regions including bilateral dorsal anterior cingulate cortex (ACC), insula, superior temporal gyrus; left frontal and parietal operculum, medial frontal gyrus, orbital prefrontal cortex, superior parietal lobule, and hippocampus; right claustrum/putamen, lateral prefrontal gyrus, and middle temporal gyrus. Functional connectivity analysis of spontaneous resting-state fMRI data from the same cohort of subjects showed a correlation between two seed regions (left frontal operculum and hippocampus) and pain network including bilateral insula, operculum, ACC, and left S1/M1. In conclusion, we found evidence that nocebo hyperalgesia may be predominantly produced through an affective-cognitive pain pathway (medial pain system), and the left hippocampus may play an important role in this process.

Key words: nocebo; nocebo effect; hyperalgesia; hyperalgesic nocebo effect; functional connectivity; spontaneous fMRI; resting state; placebo effect; expectancy; expectancy manipulation; sham acupuncture

\section{Introduction}

Placebo effects have received much attention in recent decades, and with the advent of brain imaging tools, our understanding of their neurobiology has greatly expanded (Benedetti et al., 2005; Colloca and Benedetti, 2005; Kong et al., 2007; Benedetti, 2008). In contrast, nocebo effects (adverse effects or a worsening of symptoms on account of expectation or suggestion regarding an inert treatment) have received relatively scant attention from neuroscience.

Previous studies suggest that nocebo effects, sometimes termed "negative placebo effects," contribute appreciably to a variety of medical symptoms (Barsky and Borus, 1999; Barsky et al., 2002), adverse events in clinical trials and medical care (Myers

Received June 25, 2008; revised 0ct. 22, 2008; accepted 0ct. 27, 2008.

This work was supported by National Institutes of Health-National Center for Complementary and Alternative Medicine Grants K01-AT003883 (J.K.), P01-AT002048 (B.R.), K24-AT004095 (T.J.K.), and R21-AT00949 (R.L.G.); National Center for Research Resources Grants M01-RR-01066 for Mallinckrodt General Clinical Research Center Biomedical Imaging Core and P41-RR14075 for Center for Functional Neuroimaging Technologies; and MIND Research Network (Delaware) Grant FG03-99ER62764 (B.R.).

Correspondence should be addressed to Jian Kong, Psychiatry Department, Massachusetts General Hospital, Building 149, 13th Street, Suite 2661, Charlestown, MA 02129. E-mail: kongj@nmr.mgh.harvard.edu.

DOI:10.1523/JNEUROSCI.2944-08.2008

Copyright $\odot 2008$ Society for Neuroscience ～0270-6474/08/2813354-09\$15.00/0 et al., 1987; Roscoe et al., 2000; Reuter et al., 2003; Kaptchuk et al., 2006), and public health "mass psychogenic illness" outbreaks (Jones et al., 2000). For instance, Ko et al. (2003) found patients receiving a beta blocker and patients receiving placebo report comparable levels of common side effects, including depressive symptoms, fatigue, and sexual dysfunction.

In one of the few nocebo mechanism studies to date, Benedetti et al. (1997) reported that, whereas proglumide, a nonspecific cholecystokinin (CCK) antagonist, could counteract nocebo-induced hyperalgesia, the opioid antagonist naloxone had no effect on nocebo responses. In a following study, Bendetti et al. (2006) found that, whereas the benzodiazepine diazepam could block both nocebo hyperalagesia and hypothalamic-pituitary-adrenal hyperactivity, proglumide could block only the former. As such, benzodiazepines may act on nocebo-induced anxiety, whereas proglumide may act specifically on the CCK-mediated link between pain and anxiety. An animal study (Andre et al., 2005) further documented the ability of CCK-B receptor antagonist CI-988 (4-[[2-[[3-(1H-indol-3-yl)-2methyl - 1- oxo - 2[[(tricyclo[3 .3[12,17]dec - 2 - yloxy) - carbonyl] amino]-propyl] amino]-1-phenyethyl $]$ amino $]-4$-oxo- $\left[R-\left(R^{\star}, R^{\star}\right)\right]$ butanoate $N$-methyl-D-glucamine) to block anxiety-associated hyperalgesia, suggesting hyperalgesic effects may represent an emergent property of the mechanisms linking anxiety to pain. 

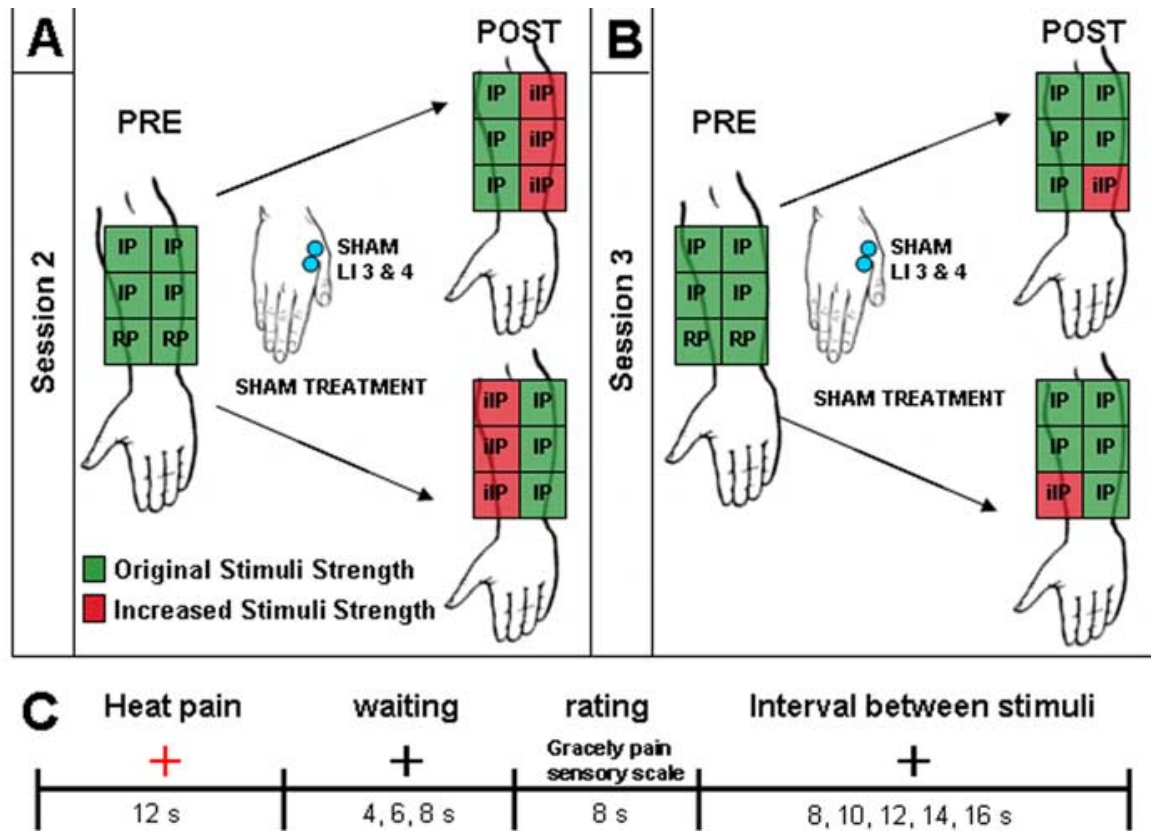

Figure 1. Experimental procedures. $\boldsymbol{A}$, In session 2, we used a marker to draw a $2 \times 3$ grid on the medial aspect of the right forearm. We placed the thermal probe in one box of the grid for each noxious stimulus sequence (RP, 8 pseudorandomized stimuli of 4 high and 4 low intensity; $I P$, 6 identical stimuli of mid intensity). After inert acupuncture (sham LI 3 and LI 4), ilP (increased IP, 6 identical stimuli of high intensity) were applied on nocebo sites, but not on control sites, giving subjects an unmistakable experience of hyperalgesia. $\boldsymbol{B}$, In session 3 , subjects were told that session 2 procedures would be repeated during fMRI scanning. However, after inert treatment, only one ilP on a nocebo site was increased (red color). The four subsequent IP on both nocebo and control sites were delivered at original, pretreatment stimulus temperatures. The differences between posttreatment and pretreatment pain ratings and fMRI signal changes applied on nocebo and control sites during the four IP were the primary outcomes of this study. C, Time course of a single trial of pain in this experiment.

Previous studies have suggested that the central pain matrix consists of approximately two parallel subsystems, the lateral (sensory-discriminatory) and medial (affective-cognitive evaluation) system (Apkarian et al., 2005; Tracey and Mantyh, 2007). The lateral system projects through the lateral thalamic nuclei to the cortex and includes the primary and secondary somatosensory cortices. The medial system projects through the medial thalamic nuclei to the cortex and includes the anterior cingulate cortex (ACC), insula, and prefrontal cortices (Price, 2000; Rainville, 2002; Vogt, 2005).

In this current experiment, we used functional magnetic resonance imaging (fMRI) and a modified expectancy/conditioning manipulation model (Montgomery and Kirsch, 1997; Price et al., 1999; Wager et al., 2004; Kong et al., 2006a) to investigate the neural substrates of hyperalgesic nocebo. At the very beginning of fMRI scanning session, we also collected a 6 min spontaneous fMRI data set to investigate functional connectivity (Raichle and Mintun, 2006; Buckner and Vincent, 2007; Fox and Raichle, 2007) among the brain regions observed to be activated in the subsequent analysis of the nocebo scans. Based on previous nocebo behavioral studies (Benedetti et al., 1997, 2006; Andre et al., 2005) indicating a role for anxiety in nocebo, we hypothesize that nocebo hyperalgesia may mainly arise from increased activation of the medial pain matrix.

\section{Materials and Methods}

Subjects

Twenty healthy, right-handed subjects, cleared for fMRI participation, enrolled in this study. To conceal the nocebo aim of the experiment, we told subjects we would be studying the effects of acupuncture on the brain. Because we wanted our intervention to be novel for patients, we used a validated sham acupuncture device and allowed only acupuncture-naive subjects to participate. Experiments were conducted with written consent of each subject and approved by the Massachusetts General Hospital Institutional Review Board. All subjects were debriefed at the end of the experiment as to the true nature of the experiment.

Procedures for the delivery and assessment of noxious thermal stimuli

Subjects participated in two behavioral testing sessions and one fMRI scanning session. Each session was separated by a minimum of $3 \mathrm{~d}$.

Calibrated thermal pain stimuli were delivered to the right medial forearm using a TSA2001 thermal sensory analyzer with a $3 \times 3 \mathrm{~cm}$ probe (Medoc Advanced Medical Systems) running proprietary computerized visual ana$\log$ scale software (COVAS). Each stimulus was initiated from a $32^{\circ} \mathrm{C}$ baseline, increased to a target temperature $\left(45.5 \sim 51^{\circ} \mathrm{C}\right)$, and presented for $12 \mathrm{~s}$, including a $2.5 \mathrm{~s}$ ramp up and ramp down. Interstimulus intervals ranged from 24 to $30 \mathrm{~s}$.

Gracely Sensory and Affective Scales (Gracely et al., 1978a,b) were used to measure subjective pain ratings. To ensure consistent pain administration, a $2 \times 3$ grid was drawn in marker along the palmar side of the forearm, with three boxes each on radial and ulnar sides. We placed the thermal probe in one box of the grid for each stimulus sequence (Fig. 1).

Session 1. We used the first behavioral session to familiarize subjects with the rating scales and determine appropriate stimulus intensities using methods used in our previous studies (Kong et al., 2005, 2006a,b). Temperatures eliciting subjective intensity ratings in the low pain range $(\sim 5$; weak on the $0-20$ sensory scale), mid pain range ( $\sim 9$; mild), and high pain range $(\sim 15$; strong) were selected for each individual and used in subsequent sessions. Next, we applied series of eight random pain noxious stimuli (four high and four low applied in random order, indicated by the abbreviation "RP") and series of six identical pain noxious stimuli (six identical mid, indicated by "IP") to the right arm. Temperatures were adjusted when necessary to ensure that each subject's subjective ratings of high, low, and mid remained in the desired range, because these would be used in the following sessions.

Session 2. We used session 2 to manipulate subjects' expectancy to sham acupuncture treatment using a method modified from our previous work (Montgomery and Kirsch, 1997; Price et al., 1999; Wager et al., 2004; Kong et al., 2006a).

At the beginning of session 2, subjects read a script stating the following: (1) responses to acupuncture can be positive, neutral, or negative, and 2) a given subject's response tends to remain consistent across sessions. Subjects then viewed a traditional Chinese medicine meridian diagram and were told the following: (3) according to previous literature, acupuncture would produce effects (positive or negative) only on the side of the arm where the meridian passed through but not on the other side of the arm. To balance the design, one-half the subjects were then shown accurate diagrams (real diagram) of the large intestine (LI) meridian passing through the radial side of the arm, whereas the other one-half viewed a modified diagram (fake diagram), showing the LI meridian passing through the ulnar side of the arm. [For clarity, we refer to meridian (real or fake) and nonmeridian (real or fake) sites as nocebo and control sites, respectively].

Next, the same RP were administered to the bottom two boxes of the $2 \times 3$ grid along the arm, and mid pain IP were applied to the top four (Fig. $1 A$ ).

To proceed in the study, subjects had to consistently rate high pain greater than low pain, and report approximately equivalent ratings $(<1.5$ 
on average intensity rating difference) to mid pain on the radial and ulnar sides of their arm.

Sham electro-acupuncture was then performed at two acupoints on the right hand (LI 4 and LI 3) using the validated, nonpenetrating Streitberger sham acupuncture device (Streitberger and Kleinhenz, 1998; Kleinhenz et al., 1999; Kong et al., 2006a; McManus et al., 2007). As in our previous methods (Kong et al., 2005), the sham needles were connected to an electro-acupuncture device (OMS Medical Supplies IC1107), with no current applied.

After treatment, we told subjects they would be receiving the same stimuli series administered before treatment. In actuality, on the nocebo side of the arm, we used an increased IP stimuli (iIP) (high instead of mid intensities), giving subjects an unmistakable experience of hypersensitivity. On the control side, IP were maintained at pretreatment mid levels (Fig. 1A).

In this session, subjects also completed an expectancy rating form ( -10 , extreme pain sensitivity; to 0 , neutral no change; to 10 , complete pain relief) once before sham treatment and once after the expectancy manipulation procedure.

Session 3. Session 3 was performed in the fMRI scanner. Subjects were told we would be repeating session 2 procedures. In actuality, whereas most procedures matched those performed in session 2, the exception was that, after treatment, only one iIP was applied on the nocebo side of the arm to boost subjects' memory of hyperalgesia, administer an additional series of the conditioning trial, and provide an experience of hyperalgesia closer in time to the nocebo test. Original mid IP stimuli were applied on all other regions of the arm. To minimize the scan time, only pain sensory rating data were collected in this session. The differences between pretreatment and posttreatment pain ratings and brain activation during these final four sequences (two mid IP on nocebo and control sides each) were the primary outcomes of this study (Fig. $1 B$ ).

At the beginning of session 3 and after the expectancy boost, subjects were required to complete the expectancy rating forms again.

\section{fMRI data acquisition and analysis}

Brain imaging was performed with a three-axis gradient head coil in a 3 tesla whole-body Thermo Fisher Scientific MRI System equipped for echoplanar imaging. Thirty axial slices ( $4 \mathrm{~mm}$ thick with $1 \mathrm{~mm}$ skip) parallel to the anterior and posterior commissure covering the whole brain were imaged with $2000 \mathrm{~ms}$ repetition time, $40 \mathrm{~ms}$ echo time, $90^{\circ}$ flip angle, and $3.13 \times 3.13 \mathrm{~mm}$ in-plane spatial resolution. A high-resolution three-dimensional magnetization-prepared rapid-acquisition gradient echo sequence was also collected for anatomic localization.

At the beginning of the scanning session, a 6 min resting status scan was performed, during which subjects were instructed to close their eyes and relax. Afterward, fMRI scanning was performed during the administration of pain (RP and IP) before and after treatment using an experimental paradigm similar to our previous studies (Kong et al., 2006a,b). During scanning, subjects were instructed to focus on a small black fixation cross in the center of a screen in front of them. The cross turned red to cue the onset and duration of each stimulus (12 s) and then turned black for a variable duration $(4,6$, or $8 \mathrm{~s})$. Then, the Sensory Box Scale was displayed on the screen $(8 \mathrm{~s})$ and subjects used a button press device controlling a pointer to indicate their subjective ratings (Fig. 1C).

\section{fMRI data analysis}

Preprocessing and statistical analyses were performed using SPM2 software (Wellcome Department of Cognitive Neurology, London, UK). Preprocessing included motion correction, normalization to MNI stereotactic space, and spatial smoothing with an $8 \mathrm{~mm}$ Gaussian kernel.

For each subject, a GLM (general linear model) design matrix was calculated, including all pain functional runs used to test nocebo effects (four runs before and after treatment on both nocebo and control sites). Then, calculations were performed on the contrast between pretreatment high pain and low pain on both nocebo and control sites. Finally, the contrast comparing post minus pre differences in response to identical IP stimuli on nocebo sites and control sites (nocebo increases relative to control) [nocebo (post - pre) - control (post - pre)] were calculated. Low-frequency noise was removed with a high-pass filter applied with default values (128s) to the fMRI time series at each voxel.
Group analysis was performed using a random-effects model. A oneway $t$ test was performed to determine group activation for each generated contrast as described above.

As in previous studies, to elucidate pain intensity-correlated brain regions with which we would later use to test for nocebo effects, we conducted an initial group comparison of all pretreatment high and low pain sequences (Wager et al., 2004; Kong et al., 2006a), constructing a mask for subsequent group analysis (with threshold set at voxelwise $p<$ 0.005 , uncorrected with 20 contiguous voxels). Based on previous studies (Petrovic and Ingvar, 2002; Benedetti et al., 2005) implicating a role for dorsal lateral prefrontal cortex (DLPFC) and orbital prefrontal cortex (OPFC) in pain modulation, these two regions were also included as a priori regions of interest (ROIs). Next, to elucidate the brain regions involved in nocebo effect, the contrast comparing post minus pre differences in response to application of IP stimuli on nocebo and control sites was calculated.

In a second level analysis, a simple regression (correlation) analysis between each subject's fMRI signal changes [nocebo (post - pre) control (post - pre)] and corresponding subjective pain rating changes was also calculated.

The threshold for predefined ROIs (either within the mask or DLPFC and OPFC) was set at voxelwise $p<0.005$ uncorrected with 20 contiguous voxels. A threshold of voxelwise $p<0.05$ corrected for five contiguous voxels was used for activation in other regions.

\section{Functional connectivity analysis}

To further investigate the functional correlation between regions, two brain regions observed in the fMRI analysis were used as seed regions for a functional connectivity study on the resting status data collected at the beginning of fMRI scan session. Methods for functional connectivity analysis were adopted from previous studies (Fox et al., 2005; AndrewsHanna et al., 2007; Vincent et al., 2007).

In summary, functional data were first preprocessed to decrease image artifacts and between-slice timing differences, and to eliminate differences in odd/even slice intensity. Rigid body translation and rotation was used to reduce within and across-run head movement. Data were resampled to $2 \mathrm{~mm}$ isotropic voxels after transforming anatomical and functional data to atlas space.

The functional connectivity analysis required additional filtering of low- and high-frequency components $(0.009 \mathrm{~Hz}<f<0.083 \mathrm{~Hz})$ and spatial $8 \mathrm{~mm}$ Gaussian kernel smoothing. Other variables that were simultaneously regressed included movement parameters, whole-brain signal, lateral ventricle mean signals, deep white matter ROI signal, and the first temporal derivative of each time course. A resulting time course was used in the subsequent analysis.

Then, correlation maps between seed regions (left operculum and left hippocampus) and all voxels across the whole brain were performed. Analysis produced seed region-whole brain voxel correlation coefficients. Fisher's $r$-to- $z$ transformation was used to convert correlation maps into $z$ maps. Group effects were tested with a random-effect analyses using one-sample $t$ test. The threshold was set at voxelwise $p<0.001$ uncorrected for 20 contiguous voxels.

\section{Results}

\section{Subjects}

Thirteen of 20 consenting volunteers completed this study (five males; age, mean \pm SD, $26.3 \pm 3.6$ years). Six subjects were dropped on account of their unreliable high and low pain ratings, unbalanced pain perceptions on ulnar and radial sides of the arm, or anxiety regarding scanning before beginning session 3. Fourteen subjects completed the scan session. Data from one scanned subject were excluded on account of excessive head movement during scanning (exceeding $5 \mathrm{~mm}$ in two functional runs).

\section{Subjective ratings of pain and expectancy}

We used session 3 pretreatment and posttreatment pain sensory intensity rating differences between nocebo and control sites to detect nocebo hyperalgesia. Pre and post ratings for identical pain 
stimuli (mean \pm SD) were calculated at $9.0 \pm 2.5$ and $11.4 \pm 2.1$ on nocebo sites, and $8.7 \pm 2.8$ and $10.1 \pm 2.1$ on control sites. The nonparametric test (Wilcoxon's signed ranks test) was used to compare the pretreatment pain ratings and pain rating changes (post minus pre) after treatment between nocebo and control sites. The results indicated that there is no difference in pain ratings between the nocebo and control sites $(p=0.33)$ before treatment. After treatment, subjective pain rating increases on the nocebo sites were significantly greater than those on the control sites $(p=0.021)$.

On the expectation scale, the subjects' expectancy ratings (mean \pm SD) significantly decreased from initially positive levels before manipulation $(2.6 \pm 2.7)$ to negative levels after manipulation $(-5.1 \pm 2.0)$ with all subjects reporting a negative expectation of the effect of acupuncture on pain in session 2 (Wilcoxon's signed rank test, $p=0.002$ ). When entering session 3, subjects' expectation ratings were maintained at significantly negative levels compared with positive expectancy ratings before manipulation in session 2 . The expectancy ratings were $-4.8 \pm$ 2.2 at the beginning of session $3(p=0.002)$ and $-5.3 \pm 1.8$ after the session 3 "expectancy boost" $(p=0.002)$.

\section{fMRI results}

The comparison between all pretreatment high and low pain stimuli (high pain $>$ low pain) applied during the RP sequence before treatment yielded significant activations in the entire predicted network of pain-sensitive regions, including bilateral insular/opercular cortices, dorsal anterior cingulate cortex (dACC), superior parietal lobule, superior and middle frontal gyrus, superior and middle temporal gyrus, hippocampus, thalamus, caudate, cerebellum, brainstem (periaqueductal gray and pons); left (contralateral) SI/M1 corresponding to the arm, medial frontal gyrus, inferior parietal lobule; and right inferior frontal gyrus, parahippocampus, angular gyrus, and precuneus (Fig. $2 A$ ). This result is consistent with previous studies (Wager et al., 2004; Kong et al., 2006a,b) and corresponds to the reported subjective pain ratings $(12.9 \pm 2.3$ and $5.4 \pm 2.8$ for high and low pain, respectively). The result of this comparison is used as a mask for the following group analysis.

When we calculated the fMRI signal contrast between posttreatment and pretreatment pain application on nocebo sites from the same difference on control sites [e.g., nocebo (post pre) - control (post - pre)], significant brain activation changes were observed in bilateral dorsal ACC, insula, superior temporal gyrus; left frontal and parietal operculum, medial frontal gyrus, orbital prefrontal cortex, superior parietal lobule, and hippocampus; right claustrum/putamen, lateral prefrontal gyrus, and middle temporal gyrus (Table 1 ). Figure $2 B$ presents representative brain regions and corresponding cluster averaged $\beta$ values for IP noxious stimuli applied before and after treatment on nocebo and control sites, as well as for low and high pain stimuli applied during the pretreatment RP sequence. As indicated in the figure, pretreatment RP high stimuli produced significantly greater fMRI signal increases than RP low stimuli at these cluster locations. In response to identical temperature posttreatment IP pain stimuli, all fMRI signals increased on nocebo sites and decreased on control sites in these brain regions.

When we calculated the contrast by subtracting fMRI signal differences between posttreatment and pretreatment control sites from the same difference on nocebo sites [e.g., control (post - pre) - nocebo (post - pre)], no brain regions passed the significance threshold.

Table 2 presents the results of a simple regression analysis between each subject's pain rating differences and corresponding fMRI signal difference between posttreatment and pretreatment differences on nocebo and control sites [e.g., nocebo (post pre) - control (post - pre)]. Significant positive correlations were observed in bilateral insula/frontal operculum and left M1. Significant negative correlations were observed in bilateral DLPFC and left OPFC.

\section{Spontaneous resting-state fMRI result}

In this study, we used ROIs created from the left frontal operculum activation and left hippocampus activation as seed regions for the analysis of functional connectivity in the spontaneous, resting-state fMRI scan collected before the start of the nocebo experimental procedures. We chose left frontal operculum because it positively correlated with subjective pain rating changes and showed significantly greater fMRI signal changes on nocebo sites compared with control sites in fMRI group analysis. (Please note the exact locations in these two analyses overlapped.) We selected the left hippocampus as a seed region because it (1) showed significant differences in group analysis; (2) played an important role in the correlation between anxiety and pain intensity ratings, as indicated by a previous study (Ploghaus et al., 2001); (3) has not been reported in the substantial placebo analgesia neuroimaging literature base (Petrovic et al., 2002; Lieberman et al., 2004; Wager et al., 2004, 2007); Zubieta et al., 2005; Bingel et al., 2006; Kong et al., 2006a; Craggs et al., 2007; Price et al., 2007; Scott et al., 2007, 2008) and may thus be uniquely involved in nocebo hyperalgesia.

Of the 13 subjects constituting the fMRI group analysis, resting status data were available for only 12 ; one subject's data were unavailable because of a technical scanning problem. To address this discrepancy in subject number, the same behavioral and fMRI group analyses were rerun using the 12 subject cohort. Identical results were found showing significant pretreatment and posttreatment subjective pain rating differences between nocebo and control sites (Wilcoxon's signed ranks test, $p<0.037$ ). fMRI analysis showed both left frontal operculum and hippocampus surviving reanalysis using the same threshold as before, whereas the cluster size for both regions decreased. Based on these calculations, we chose to use whole clusters as seed regions for spontaneous fMRI analysis of the 12 subjects. The details for these seed regions (coordinate, $z$ value, and cluster size) are as follows: left insula/operculum ( $-5028 ; z=3.10 ; 38$ voxels) and left hippocampus $(-34-18-14 ; z=3.84 ; 21$ voxels).

The results of spontaneous fMRI analysis using left insula/ operculum and hippocampus as seed regions are shown in Figure 3 and Table 3. For left operculum/insula, regions included bilateral operculum/insula/precentral and postcentral gyrus/inferior parietal lobule/inferior frontal gyrus/superior temporal gyrus/ hippocampus/putamen, bilateral ACC/medial frontal gyrus, DLPFC, brainstem, left S1/M1, and right midcingulate and posterior cingulate cortex. For left hippocampus, brain regions included bilateral hippocampus, superior, middle, and inferior temporal gyrus, insula/frontal and parietal operculum, postcentral and precentral gyrus, putamen, ACC, and right orbital prefrontal cortex.

\section{Discussion}

In this study, we found that, after invoking nocebo effects through the creation of negative expectancy to a sham treatment, subjective pain ratings (post minus pre) increased significantly more on nocebo sites of the arm compared with control sites. $\mathrm{fMRI}$ analysis showed brain regions involved in hyperalgesic no- 


\section{A. Activations during encoding of pain sensation}
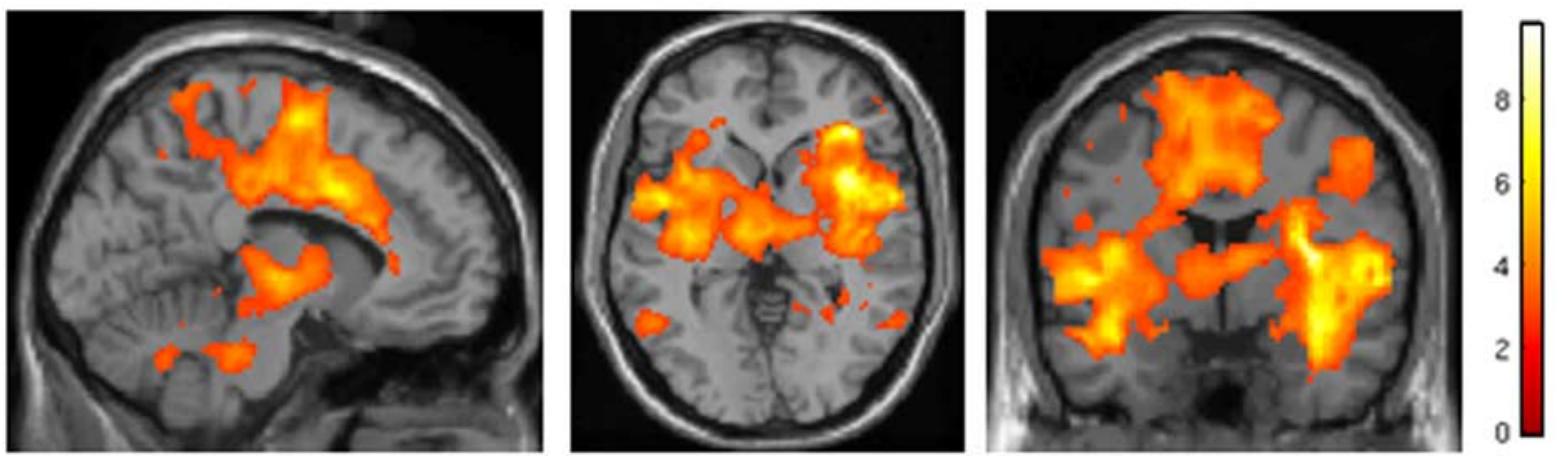

B. Representative regions involved in nocebo hyperalgesia
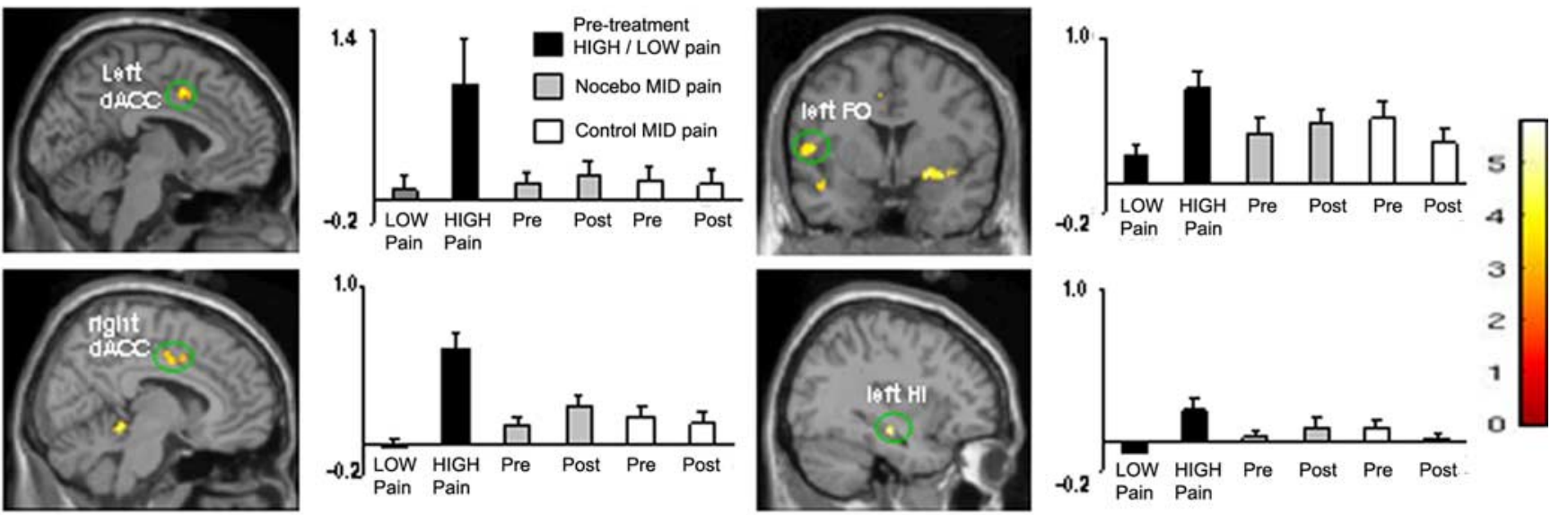

\section{Representative regions correlated with nocebo hyperalgesia}
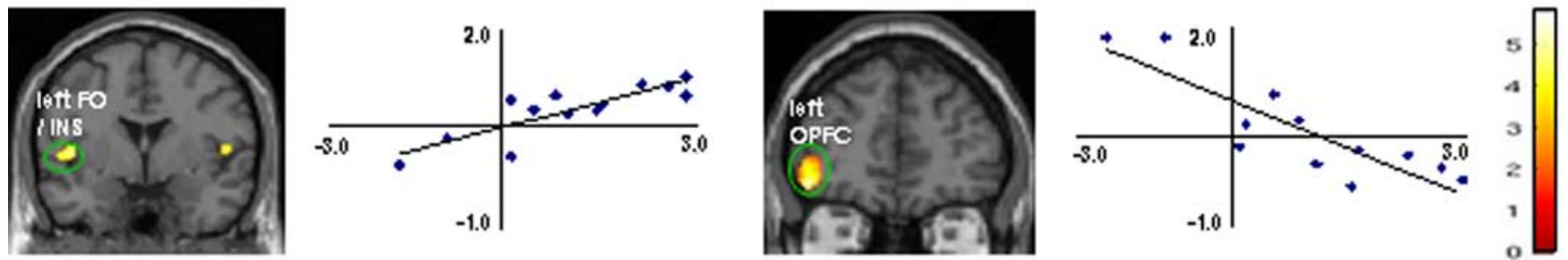

Figure 2. A, fMRI signal changes invoked by all RP pretreatment high pain stimuli minus all pretreatment low pain stimuli applied on both placebo and control sites. Brain regions including bilateral insular/opercular cortices, dACC, SII, thalamus, temporal gyrus, and left SI/M1 (contralateral), among others showed activation above the threshold. These regions were used as a mask for subsequent fMRI analyses. $\boldsymbol{B}$, Representative brain regions involved in nocebo effect. The bars indicate cluster $\beta$ values of low and high pain when RP sequences were applied, and values of mid pain when IP sequences were applied on nocebo and control sites (average \pm SD). F0, Frontal operculum; Hi, hippocampus. C, Representative regions showing significant correlations with behavioral results. The $x$-axis represents the difference between posttreatment and pretreatment subjective pain ratings between nocebo and control sites. The $y$-axis represents peak $\beta$ values of fMRI signal change. FO/INS, Frontal operculum/insula.

cebo effect during pain administration to include bilateral dorsal ACC, insula, superior temporal gyrus; left frontal and parietal operculum, medial frontal gyrus, orbital prefrontal cortex, superior parietal lobule, and hippocampus; right claustrum/putamen, lateral prefrontal gyrus, and middle temporal gyrus. Additional analysis of spontaneous fMRI data collected before pain application showed functional connections among left frontal operculum and hippocampus and the brain regions belonging to the pain network, including bilateral insula, operculum, ACC, and left M1.

We found the brain regions preferentially activated during nocebo hyperalgesic pain administration, including bilateral ACC, insula, left orbital frontal cortex, and right lateral prefrontal cortex, to reside primarily in the medial system of the pain ma- trix. This result is consistent with our hypothesis that nocebo hyperalgesia is predominantly produced though the affectivecognitive pain pathway. Previous studies have characterized the behavioral response to nocebo hyperalgesia and described the important role of anxiety in this process (Benedetti et al., 2006). We believe our work is the first to elucidate the brain network underlying nocebo hyperalgesia.

Previous brain imaging studies have indicated that expectation can significantly modulate subsequent noxious stimuli perception (Sawamoto et al., 2000; Koyama et al., 2005; Keltner et al., 2006). For instance, Sawamoto et al. (2000) found that uncertain expectation regarding impending painful stimuli could enhance brain responses to nonpainful stimuli, increasing the intensity and range of activation in the ACC and parietal operculum and posterior insula, respec- 
Table 1. Results of a paired $t$ test comparing fMRI signal change differences (posttreatment pain > pretreatment pain) between nocebo and control sites

\begin{tabular}{|c|c|c|c|c|c|}
\hline \multicolumn{2}{|c|}{ fMRI signal change differences } & \multirow{2}{*}{$\begin{array}{l}\text { Area (Brodmann area) } \\
\text { Left dACC (24) }\end{array}$} & \multirow{2}{*}{$\frac{Z \text { score }}{3.51}$} & \multirow{2}{*}{$\begin{array}{l}\text { No. of voxels in cluster } \\
72\end{array}$} & \multirow{2}{*}{$\begin{array}{l}\text { Peak coordinate }(x, y, z) \\
-4,6,44\end{array}$} \\
\hline Nocebo $>$ control & Within mask and predefined ROIs & & & & \\
\hline & & Right dACC (24) & 3.40 & 104 & $10,-6,46$ \\
\hline & & Left frontal operculum & 3.34 & 71 & $-50,2,8$ \\
\hline & & Left insula/parietal operculum & 2.90 & 36 & $-44,-28,18$ \\
\hline & & Right putamen/claustrum/insula & 4.01 & 256 & $34,-16,2$ \\
\hline & & Left superior temporal gyrus/insula (38/21) & 3.72 & 61 & $-46,0,-16$ \\
\hline & & Left hippocampus & 3.78 & 25 & $-34,-18,-14$ \\
\hline & & Left medial frontal gyrus (6) & 3.09 & 22 & $-10,-4,60$ \\
\hline & & Left superior parietal lobule (7) & 2.97 & 23 & $-14,-46,64$ \\
\hline & & Left superior temporal gyrus (22) & 3.10 & 27 & $-62,-30,14$ \\
\hline & & Right superior/middle temporal gyrus (22/21) & 3.36 & 43 & $56,-56,8$ \\
\hline & & Right lateral prefrontal gyrus (44/9) & 3.77 & 24 & $32,10,30$ \\
\hline & & Left orbital prefrontal gyrus (47) & 3.49 & 27 & $-36,30,-10$ \\
\hline & Others & No regions above the threshold & & & \\
\hline Control > nocebo & & No regions above the threshold & & & \\
\hline
\end{tabular}

The threshold for brain regions located within the mask and predefined R0ls was set to voxelwise $p<0.005$ uncorrected with 20 continuous voxels, and voxelwise $p<0.05$ corrected for 5 contiguous voxels for other regions. Italics indicate regions within the high pain minus low pain mask. Peak coordinates refer to the Montreal Neurological Institute atlas.

Table 2. Regression (correlation) analysis between each subject's fMRI signal changes [nocebo (post - pre) - control (post - pre)] and corresponding subjective pain rating changes

\begin{tabular}{|c|c|c|c|c|c|c|}
\hline Correlation & & Area (Brodmann area) & Zscore & No. of voxels in cluster & Peak $r$ value & Peak coordinate $(x, y, z)$ \\
\hline \multirow[t]{4}{*}{+} & Within mask and ROls & Left M1 (4) & 3.75 & 20 & 0.86 & $-20,-18,54$ \\
\hline & & Left insula/frontal operculum & 3.74 & 66 & 0.86 & $-44,0,6$ \\
\hline & & Right insula/frontal operculum & 3.51 & 37 & 0.83 & $50,-2,4$ \\
\hline & 0thers & No regions above threshold & & & & \\
\hline \multirow[t]{7}{*}{ - } & Within mask ROls & Left lateral OPFC (47/11) & 4.52 & 299 & -0.88 & $-44,46,-10$ \\
\hline & & Left DLPFC (9) & 4.40 & 863 & -0.91 & $-46,4,34$ \\
\hline & & Right DLPFC (9) & 4.37 & 263 & -0.91 & $38,10,42$ \\
\hline & & Left medial OPFC (47/11) & 3.77 & 115 & -0.85 & $-14,32,-12$ \\
\hline & & Right DLPFC (46) & 3.32 & 52 & -0.80 & $46,36,16$ \\
\hline & & Left DLPFC (8) & 3.17 & 86 & -0.71 & $-20,30,56$ \\
\hline & Others & No regions above the threshold & & & & \\
\hline
\end{tabular}

The threshold for predefined ROIs was set to voxelwise $p<0.005$ uncorrected with 20 continuous voxels, and voxelwise $p<0.05$ corrected for 5 contiguous voxels for other regions. Italics indicate regions within the high pain minus low pain mask. Peak coordinates refer to the Montreal Neurological Institute atlas.
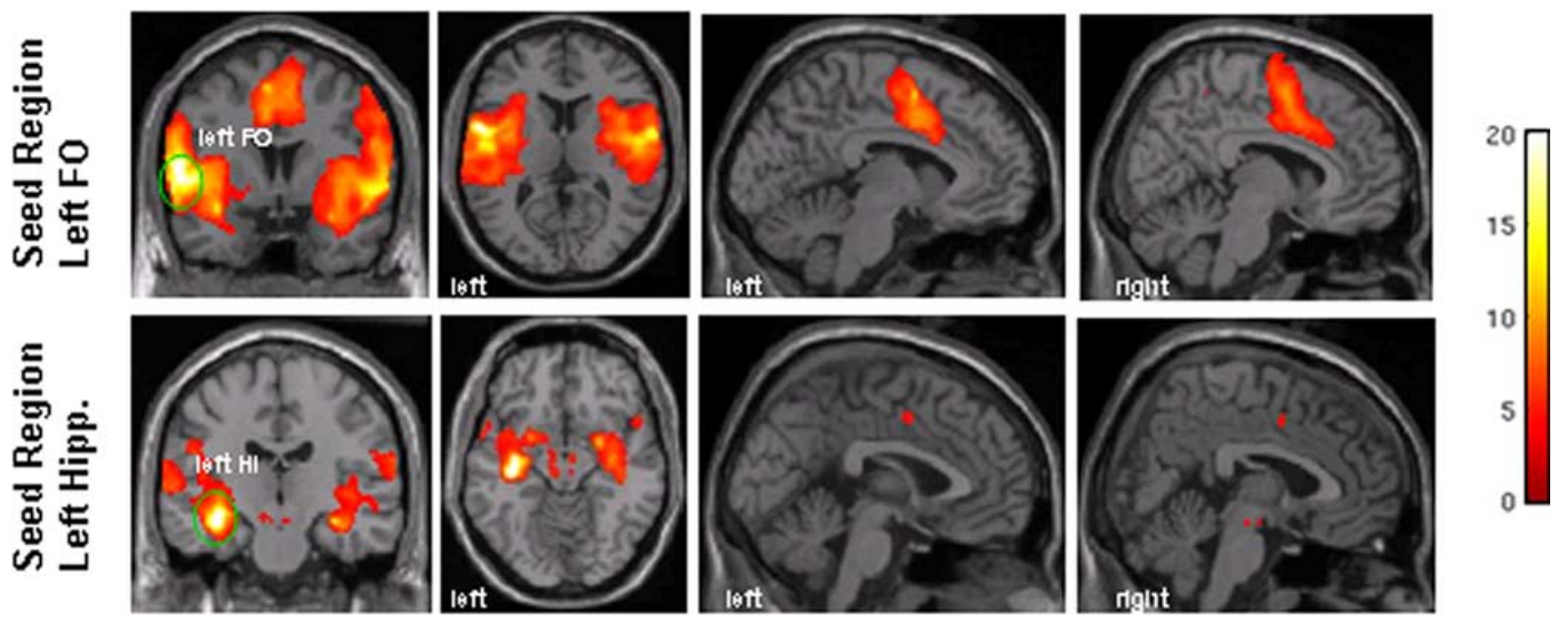

Figure 3. Brain regions observed in the functional connectivity analysis using left frontal operculum (FO) and hippocampus (Hipp.; HI) as seed regions. The threshold was set to voxelwise $p<$ 0.001 uncorrected with 20 contiguous voxels.

tively. In a later study, Keltner et al. (2006) found that expectancy to higher levels of pain could significantly increase reported pain intensity ratings and enhance activation of afferent pain circuits in the ipsilateral ACC, caudate, cerebellum, and contralateral nucleus cuneiformis. They further hypothesized that facilitation of the de- scending pain modulation pathway may be involved in this process. Although not completely the same, these studies are partly consistent with our finding that, during nocebo hyperalgesia, brain activity in bilateral ACC, insula, and operculum on nocebo sites increases significantly more than on control sites. 
Table 3. Results of functional connectivity on resting status data showing brain regions correlated with seed regions

\begin{tabular}{|c|c|c|c|c|}
\hline Seed regions & Area (Brodmann area) & Zscore & No. of voxels in cluster & Peak coordinate $(x, y, z)$ \\
\hline \multirow[t]{7}{*}{ Left F0 } & $\begin{array}{l}\text { Left operculum/insula/precentral and postcentral gyrus/inferior } \\
\text { parietal lobule/ inferior frontal gyrus/superior temporal gy- } \\
\text { rus/hippocampus/putamen }(1,2,3,4,6,40,44,21,22)\end{array}$ & 7.28 & 9941 & $-56,4,10$ \\
\hline & $\begin{array}{l}\text { Right operculum/insula/precentral and postcentral gyrus/infe- } \\
\text { rior parietal lobule/superior temporal gyrus/hippocampus/ } \\
\text { putamen/brainstem }(1,2,3,4,6,40,44,21,22)\end{array}$ & 5.87 & 11,645 & $48,-14,20$ \\
\hline & $\begin{array}{l}\text { Bilateral ACC/medial frontal gyrus/right midcingulate and } \\
\text { postcingulate cortex }(24,23,31)\end{array}$ & 5.16 & 3521 & $-4,4,54$ \\
\hline & Left DLPFC $(46,9)$ & 4.14 & 117 & $-30,36,24$ \\
\hline & Right DLPFC $(46,9)$ & 3.72 & 47 & $46,48,28$ \\
\hline & Left S1/M1 $(4,3)$ & 3.58 & 37 & $-26,-30,78$ \\
\hline & Left brainstem & 3.49 & 22 & $-10,-18,-6$ \\
\hline \multirow[t]{11}{*}{ Left Hi } & $\begin{array}{l}\text { Left hippocampus/superior and middle and inferior temporal } \\
\text { gyrus/insula/frontal and parietal operculum/postcentral and } \\
\text { precentral gyrus/putamen/brainstem }(21,20,38,1,2,3,4,6)\end{array}$ & 7.55 & 6157 & $-34,-18,-12$ \\
\hline & $\begin{array}{l}\text { Right hippocampus/superior and middle temporal gyrus/insula/ } \\
\text { frontal and parietal operculum/postcentral and precentral } \\
\text { gyrus/putamen }\end{array}$ & 6.47 & 4746 & $36,-14,-16$ \\
\hline & Left middle/inferior temporal gyrus $(20,38)$ & 5.54 & 302 & $38,-2,-40$ \\
\hline & Right postcentral gyrus/superior parietal lobule $(2,5)$ & 4.15 & 23 & $-16,-46,80$ \\
\hline & Left postcentral gyrus/superior parietal lobule $(2,5)$ & 4.00 & 46 & $-18,-44,80$ \\
\hline & Right insula/putamen & 3.99 & 130 & $34,24,6$ \\
\hline & Right ACC $(32,24)$ & 3.87 & 29 & $16,8,42$ \\
\hline & Right precentral gyrus $(4,6)$ & 3.74 & 32 & $54,-4,50$ \\
\hline & Right orbital prefrontal gyrus (47) & 3.54 & 29 & $40,22,-18$ \\
\hline & Bilateral ACC (24) & 3.53 & 35 & $2,0,46$ \\
\hline & Right brainstem & 3.4 & 21 & $10,-34,-46$ \\
\hline
\end{tabular}

The threshold was set to voxelwise $p<0.001$ with 20 continuous voxels. Peak coordinates refer to the Montreal Neurological Institute atlas. F0, Frontal operculum; Hi, hippocampus.

Recently, spontaneous brain activity has been used to investigate functional connectivity among different brain regions (Raichle and Mintun, 2006; Buckner and Vincent, 2007; Fox and Raichle, 2007). In an early study, Biswal et al. (1995) found that, while subjects were at rest, spontaneous fMRI blood oxygen leveldependent signal fluctuations observed in left sensory motor areas showed a high degree of temporal correlation with right sensory motor-related brain areas and medial motor areas. This study has been replicated and findings extended to many other brain systems (Fox and Raichle, 2007). Consistent with these findings, we found highly symmetric left and right side connectivity correlations for both seed regions. Furthermore, in addition to the brain regions surrounding each seed, many regions observed in this analysis belonged to the pain network, suggesting that connectivity persists in the absence of pain stimuli as well.

In this experiment, we observed left frontal operculum activation in both group analysis and regression analysis, indicating the role of this region in nocebo hyperalgesia. Previous studies implicate operculum/insula as the most reliable region in brain imaging studies on pain (Peyron et al., 2000) and report its direct association with S1, SII, prefrontal areas, superior temporal gyrus, amygdaloid, and perirhinal cortex, which is an important source of hippocampal and ACC afferents (Augustine, 1996; Cipolloni and Pandya, 1999). The above connections link brain regions to the somatosensory, limbic/paralimbic, and working memory systems, providing an anatomic basis for the multiple functions and extensive functional connectivity observed in spontaneous fMRI data.

Our study also showed nocebo-induced fMRI signal changes in left hippocampus, a region known to play an important role in encoding relations between various learning context cues (Olsson and Phelps, 2007) and mediating aversive drive and the af- fective characteristics of pain (Melzack and Casey, 1968). The left hippocampus has been previously reported in fMRI studies on pain and anxiety. In one such study, Ploghaus et al. (2001) investigated brain response to identical pain at varying anxiety levels, observing a relationship between greater anxiety and higher pain intensity ratings. They also found left hippocampus to be uniquely involved in this process and reported that, during anxiety-induced emotional pain modulation, hippocampal responses can predict activity in closely connected, affective (perigenual cingulate), and intensity coding (midinsula) areas. The study by Ploghaus et al. (2001) indicates that, during states of heightened anxiety, the hippocampus can amplify aversive events so as to prime behavioral responses that are adaptive for dealing with the worst possible outcome. We speculate similar mechanism may also underlie nocebo hyperalgesia.

To further evaluate the role of the hippocampus in hyperalgesic nocebo, we performed a correlation analysis between subjects' cluster $\beta$ values for left hippocampus and other brain regions as shown in Table 1 . We found that left orbital prefrontal gyrus $(p=0.003 ; r=0.76)$ and right dACC $(p=0.024 ; r=0.62)$ were significantly correlated with left hippocampus brain activity. The lateral orbital prefrontal cortex and dACC are known to play key roles in cognitively modulating the emotional components of pain (Petrovic and Ingvar, 2002) and processing affective aspects of pain (Price, 2000), respectively.

Interestingly, although the brain-imaging literature for placebo analgesia is quite robust (Petrovic et al., 2002; Lieberman et al., 2004; Wager et al., 2004, 2007; Zubieta et al., 2005; Bingel et al., 2006; Kong et al., 2006a; Craggs et al., 2007; Price et al., 2007; Scott et al., 2007, 2008), no placebo analgesia study to date has ever reported involvement of the hippocampus. Functional connectivity analyses using the hippocampus as a seed region dem- 
onstrate its widespread connection to pain matrix brain regions, including bilateral insula/operculum, ACC, superior parietal lobule, left M1, and premotor areas. This result provides additional support for a possible unique role of the hippocampus in mediating nocebo hyperalgesia compared with placebo analgesia.

In this experiment, we found a significant fMRI signal increase to pain in bilateral ACC, a key region involved in processing the affective components of pain (Rainville et al., 1997; Price, 2000; Rainville, 2002). Interestingly, when we performed the functional connectivity analysis using left frontal operculum and hippocampus as seed regions, bilateral ACC activity was observed in both cases. Signal patterns were adjacent or overlapped with fMRI group analysis results (Figs. 2, 3). These results further support the role of affective processing in nocebo hyperalgesia.

Our study found activity in left orbital prefrontal cortex (PFC) and right DLPFC for fMRI group analysis. A negative correlation between fMRI signal change and subjective ratings was also observed in bilateral DLPFC and left OPFC. We speculate that activity changes in PFC and parietal lobule may imply multiple functions, including memory retrieval of previous experience, expectation generation, modulation of pain perception and pain ratings, as well as attention and emotion modulation (Benedetti et al., 2006; Kong et al., 2007).

In a recent positron emission tomography study, Scott et al. (2008) asked subjects to undergo a $20 \mathrm{~min}$ pain challenge and found placebo-enhanced opioid neurotransmission in the anterior cingulate, orbitofrontal and insular cortex, nucleus accumbens, amygdala, and periaqueductal gray, as well as dopamine activation (DA) in the ventral basal ganglia, including the nucleus accumbens. In the same study, five subjects responding negatively to the pain challenge showed opposite changes in brain activity, a deactivation of DA, and decreased opioid release in brain regions mentioned above. According to this finding, nocebo-related brain regions, including anterior cingulate, orbitofrontal and insular cortex, nucleus accumbens, and amygdala are all important limbic regions related to the interaction of emotion and pain (Vogt, 2005). Although the nature of this study may differ from our own [please note that, in the study by Scott et al. (2008), which we believed was originally designed to test placebo effect, subjects were told they would receive either an active analgesic drug or placebo, and thus the relationship of these findings to nocebo effects may be limited], we similarly found nocebo hyperalgesia to exert its effects through the affective component of pain network. In addition, our work also further indicates a more extensive network of brain regions involved in nocebo hyperalgesia, including the lateral prefrontal cortex, parietal lobule, and left hippocampus.

In conclusion, we found evidence that the nocebo hyperalgesic effect may be produced through the medial system of the central pain matrix responsible for affective/emotional and cognitive aspects of pain perception. Analysis of spontaneous fMRI data, collected in the absence of and preceding any pain stimuli, showed a functional connection among the brain regions observed in the subsequent nocebo scans. The left hippocampus may play an important role in nocebo hyperalgesia.

\section{References}

Andre J, Zeau B, Pohl M, Cesselin F, Benoliel JJ, Becker C (2005) Involvement of cholecystokininergic systems in anxiety-induced hyperalgesia in male rats: behavioral and biochemical studies. J Neurosci 25:7896-7904. Andrews-Hanna JR, Snyder AZ, Vincent JL, Lustig C, Head D, Raichle ME, Buckner RL (2007) Disruption of large-scale brain systems in advanced aging. Neuron 56:924-935.

Apkarian AV, Bushnell MC, Treede RD, Zubieta JK (2005) Human brain mechanisms of pain perception and regulation in health and disease. Eur J Pain 9:463-484.

Augustine JR (1996) Circuitry and functional aspects of the insular lobe in primates including humans. Brain Res Brain Res Rev 22:229-244.

Barsky AJ, Borus JF (1999) Functional somatic syndromes. Ann Intern Med 130:910-921.

Barsky AJ, Saintfort R, Rogers MP, Borus JF (2002) Nonspecific medication side effects and the nocebo phenomenon. JAMA 287:622-627.

Benedetti F (2008) Mechanisms of placebo and placebo-related effects across diseases and treatments. Annu Rev Pharmacol Toxicol 48:33-60.

Benedetti F, Amanzio M, Casadio C, Oliaro A, Maggi G (1997) Blockade of nocebo hyperalgesia by the cholecystokinin antagonist proglumide. Pain 71:135-140.

Benedetti F, Mayberg HS, Wager TD, Stohler CS, Zubieta JK (2005) Neurobiological mechanisms of the placebo effect. J Neurosci 25:10390-10402.

Benedetti F, Amanzio M, Vighetti S, Asteggiano G (2006) The biochemical and neuroendocrine bases of the hyperalgesic nocebo effect. J Neurosci 26:12014-12022.

Bingel U, Lorenz J, Schoell E, Weiller C, Büchel C (2006) Mechanisms of placebo analgesia: rACC recruitment of a subcortical antinociceptive network. Pain 120:8-15.

Biswal B, Yetkin FZ, Haughton VM, Hyde JS (1995) Functional connectivity in the motor cortex of resting human brain using echo-planar MRI. Magn Reson Med 34:537-541.

Buckner RL, Vincent JL (2007) Unrest at rest: default activity and spontaneous network correlations. Neuroimage 37:1091-1096; discussion 1097-1099.

Cipolloni PB, Pandya DN (1999) Cortical connections of the frontoparietal opercular areas in the rhesus monkey. J Comp Neurol 403:431-458.

Colloca L, Benedetti F (2005) Placebos and painkillers: is mind as real as matter? Nat Rev Neurosci 6:545-552.

Craggs JG, Price DD, Verne GN, Perlstein WM, Robinson MM (2007) Functional brain interactions that serve cognitive-affective processing during pain and placebo analgesia. Neuroimage 38:720-729.

Fox MD, Raichle ME (2007) Spontaneous fluctuations in brain activity observed with functional magnetic resonance imaging. Nat Rev Neurosci 8:700-711.

Fox MD, Snyder AZ, Vincent JL, Corbetta M, Van Essen DC, Raichle ME (2005) The human brain is intrinsically organized into dynamic, anticorrelated functional networks. Proc Natl Acad Sci U S A 102:9673-9678.

Gracely RH, McGrath F, Dubner R (1978a) Ratio scales of sensory and affective verbal pain descriptors. Pain 5:5-18.

Gracely RH, McGrath P, Dubner R (1978b) Validity and sensitivity of ratio scales of sensory and affective verbal pain descriptors: manipulation of affect by diazepam. Pain 5:19-29.

Jones TF, Craig AS, Hoy D, Gunter EW, Ashley DL, Barr DB, Brock JW, Schaffner W (2000) Mass psychogenic illness attributed to toxic exposure at a high school. N Engl J Med 342:96-100.

Kaptchuk TJ, Stason WB, Davis RB, Legedza AR, Schnyer RN, Kerr CE, Stone DA, Nam BH, Kirsch I, Goldman RH (2006) Sham device v inert pill: randomised controlled trial of two placebo treatments. BMJ 332:391-397.

Keltner JR, Furst A, Fan C, Redfern R, Inglis B, Fields HL (2006) Isolating the modulatory effect of expectation on pain transmission: a functional magnetic resonance imaging study. J Neurosci 26:4437-4443.

Kleinhenz J, Streitberger K, Windeler J, Güssbacher A, Mavridis G, Martin E (1999) Randomised clinical trial comparing the effects of acupuncture and a newly designed placebo needle in rotator cuff tendinitis. Pain 83:235-241.

Ko DT, Hebert PR, Krumholz HM (2003) Review: Beta-blockers increase fatigue and sexual dysfunction but not depression after myocardial infarction. ACP J Club 138:30; author reply 30.

Kong J, Fufa DT, Gerber AJ, Rosman IS, Vangel MG, Gracely RH, Gollub RL (2005) Psychophysical outcomes from a randomized pilot study of manual, electro, and sham acupuncture treatment on experimentally induced thermal pain. J Pain 6:55-64.

Kong J, Gollub RL, Rosman IS, Webb JM, Vangel MG, Kirsch I, Kaptchuk TJ (2006a) Brain activity associated with expectancy-enhanced placebo analgesia as measured by functional magnetic resonance imaging. J Neurosci 26:381-388.

Kong J, White NS, Kwong KK, Vangel MG, Rosman IS, Gracely RH, Gollub RL (2006b) Using fMRI to dissociate sensory encoding from cognitive evaluation of heat pain intensity. Hum Brain Mapp 27:715-721. 
Kong J, Kaptchuk TJ, Polich G, Kirsch I, Gollub RL (2007) Placebo analgesia: findings from brain imaging studies and emerging hypotheses. Rev Neurosci 18:173-190.

Koyama T, McHaffie JG, Laurienti PJ, Coghill RC (2005) The subjective experience of pain: where expectations become reality. Proc Natl Acad Sci U S A 102:12950-12955.

Lieberman MD, Jarcho JM, Berman S, Naliboff BD, Suyenobu BY, Mandelkern M, Mayer EA (2004) The neural correlates of placebo effects: a disruption account. Neuroimage 22:447-455.

McManus CA, Schnyer RN, Kong J, Nguyen LT, Hyun Nam B, Goldman R, Stason WB, Kaptchuk TJ (2007) Sham acupuncture devices: practical advice for researchers. Acupunct Med 25:36-40.

Melzack R, Casey KL (1968) Sensory, motivational, and central control determinants of pain. In: The skin senses (Kenshalo DR, ed). Springfield, IL: Charles C. Thomas.

Montgomery GH, Kirsch I (1997) Classical conditioning and the placebo effect. Pain 72:107-113.

Myers MG, Cairns JA, Singer J (1987) The consent form as a possible cause of side effects. Clin Pharmacol Ther 42:250-253.

Olsson A, Phelps EA (2007) Social learning of fear. Nat Neurosci 10:1095-1102.

Petrovic P, Ingvar M (2002) Imaging cognitive modulation of pain processing. Pain 95:1-5.

Petrovic P, Kalso E, Petersson KM, Ingvar M (2002) Placebo and opioid analgesia: imaging a shared neuronal network. Science 295:1737-1740.

Peyron R, Laurent B, García-Larrea L (2000) Functional imaging of brain responses to pain. A review and meta-analysis (2000). Neurophysiol Clin 30:263-288.

Ploghaus A, Narain C, Beckmann CF, Clare S, Bantick S, Wise R, Matthews PM, Rawlins JN, Tracey I (2001) Exacerbation of pain by anxiety is associated with activity in a hippocampal network. J Neurosci 21:9896-9903.

Price DD (2000) Psychological and neural mechanisms of the affective dimension of pain. Science 288:1769-1772.

Price DD, Milling LS, Kirsch I, Duff A, Montgomery GH, Nicholls SS (1999) An analysis of factors that contribute to the magnitude of placebo analgesia in an experimental paradigm. Pain 83:147-156.

Price DD, Craggs J, Verne GN, Perlstein WM, Robinson ME (2007) Placebo analgesia is accompanied by large reductions in pain-related brain activity in irritable bowel syndrome patients. Pain 127:63-72.
Raichle ME, Mintun MA (2006) Brain work and brain imaging. Annu Rev Neurosci 29:449-476.

Rainville P (2002) Brain mechanisms of pain affect and pain modulation. Curr Opin Neurobiol 12:195-204.

Rainville P, Duncan GH, Price DD, Carrier B, Bushnell MC (1997) Pain affect encoded in human anterior cingulate but not somatosensory cortex. Science 277:968-971.

Reuter U, Sanchez del Rio M, Carpay JA, Boes CJ, Silberstein SD (2003) Placebo adverse events in headache trials: headache as an adverse event of placebo. Cephalalgia 23:496-503.

Roscoe JA, Hickok JT, Morrow GR (2000) Patient expectations as predictor of chemotherapy-induced nausea. Ann Behav Med 22:121-126.

Sawamoto N, Honda M, Okada T, Hanakawa T, Kanda M, Fukuyama H, Konishi J, Shibasaki H (2000) Expectation of pain enhances responses to nonpainful somatosensory stimulation in the anterior cingulate cortex and parietal operculum/posterior insula: an event-related functional magnetic resonance imaging study. J Neurosci 20:7438-7445.

Scott DJ, Stohler CS, Egnatuk CM, Wang H, Koeppe RA, Zubieta JK (2007) Individual differences in reward responding explain placebo-induced expectations and effects. Neuron 55:325-336.

Scott DJ, Stohler CS, Egnatuk CM, Wang H, Koeppe RA, Zubieta JK (2008) Placebo and nocebo effects are defined by opposite opioid and dopaminergic responses. Arch Gen Psychiatry 65:220-231.

Streitberger K, Kleinhenz J (1998) Introducing a placebo needle into acupuncture research. Lancet 352:364-365.

Tracey I, Mantyh PW (2007) The cerebral signature for pain perception and its modulation. Neuron 55:377-391.

Vincent JL, Patel GH, Fox MD, Snyder AZ, Baker JT, Van Essen DC, Zempel JM, Snyder LH, Corbetta M, Raichle ME (2007) Intrinsic functional architecture in the anaesthetized monkey brain. Nature 447:83-86.

Vogt BA (2005) Pain and emotion interactions in subregions of the cingulate gyrus. Nat Rev Neurosci 6:533-544.

Wager TD, Rilling JK, Smith EE, Sokolik A, Casey KL, Davidson RJ, Kosslyn SM, Rose RM, Cohen JD (2004) Placebo-induced changes in FMRI in the anticipation and experience of pain. Science 303:1162-1167.

Wager TD, Scott DJ, Zubieta JK (2007) Placebo effects on human muopioid activity during pain. Proc Natl Acad Sci U S A 104:11056-11061.

Zubieta JK, Bueller JA, Jackson LR, Scott DJ, Xu Y, Koeppe RA, Nichols TE Stohler CS (2005) Placebo effects mediated by endogenous opioid activity on $\mu$-opioid receptors. J Neurosci 25:7754-7762. 